Sharif University of Technology
Scientia Iranica
Transactions E: Industrial Engineering
wCIENTIA

\title{
A projection-based approach to intuitionistic fuzzy group decision making
}

\author{
Z. Yue ${ }^{a, *}$ and Y. Jia ${ }^{b}$ \\ a. College of Mathematics and Computer Science, Guangdong Ocean University, Zhanjiang, China. \\ b. Library of Guangdong Ocean University, Zhanjiang, China. \\ Received 19 October 2014; received in revised form 24 April 2016; accepted 28 June 2016
}

\author{
KEYWORDS \\ Group decision \\ making; \\ Intuitionistic fuzzy \\ number; \\ Weight of decision \\ maker; \\ Projection \\ measurement; \\ Aggregation.
}

\begin{abstract}
Group Decision Making (GDM) is usually used for solving complex decision problems, which is an important part of modern decision science. Weight of the Decision Maker (DM) plays an important role in the GDM process, and the projection-based approach is a comprehensive consideration between decision objects. It is a valuable work to determine the weights of DMs by a projection measurement. This paper investigates a GDM method based on projection measurement in an intuitionistic fuzzy environment. First, this article introduces an ideal decision among all individual decisions, and the weights of DMs are determined by using a projection measurement. Then, the individual decisions are aggregated into a collective decision. Finally, the preference order of alternatives is identified by using the score and accuracy function of the intuitionistic fuzzy numbers. In addition, a comparison with another GDM method is provided. Feasibility and practicability of the developed method are illustrated by an experimental analysis. The experimental result shows that the projection-based method is a high-resolution decision method.

(C) 2017 Sharif University of Technology. All rights reserved.
\end{abstract}

\section{Introduction}

Decision making is a very common human activity. Since the real-life decision problems often involve multiple types of attributes (or criteria, or factors, or indexes), it is difficult to satisfy all attributes simultaneously in a decision problem. In other words, an alternative may satisfy some attributes whereas it may fail in satisfying other attributes. In that context, Multiple-Attribute Decision Making (MADM) [1-3] provides us with a good decision tool. MADM is used to find a desirable solution from a finite number of alternatives, in which some conflicting and noncommensurable attributes may be involved [4,5]. The MADM methods so far have been successfully applied

*. Corresponding author.

E-mail addresses: zhongliangyue@gmail.com (Z. Yue); jiayy1959@163.com (Y. Jia) to various areas [6-8]. However, due to the increasing complexity of the socio-economic environment, some decisions in the real-world situation often need to consider multiple points of view, performing Group Decision Making (GDM) [9-11] in which a group of experts provide their preferences to achieve a solution.

GDM methods have been extensively applied in various areas. For example, Chen et al. [12] introduced a GDM based on intuitionistic fuzzy sets and evidential reasoning methodology. Ebrahimnejad et al. [13] presented a novel two-phase GDM approach for construction project selection in a fuzzy environment. Vahdani et al. [14] developed a compromise solution method for fuzzy GDM problems with an application in the contractor selection. Mousavi et al. [15] offered a fuzzy stochastic GDM approach for selection problems. Vahdani et al. [16] suggested a GDM method based on a novel technique. Mousavi et al. [17] presented a soft computing method based on a fuzzy grey group 
compromise solution. Mousavi et al. [18] developed a hierarchical GDM approach for new product selection in a fuzzy environment. Sadr et al. [19] proposed a GDM tool for the application of membrane technologies in different water reuse scenarios. Fasanghari et al. [20] developed a novel credibility-based GDM method for enterprise architecture scenario analysis using data envelopment analysis. Aloini et al. [21] proposed a decision support system for packaging machine selection. Merigo' et al. [22] introduced the uncertain generalized probabilistic weighted averaging operator for GDM. Feng and Lai [23] proposed an integrated GDM approach with consideration of Decision Maker's (DM's) or expert's aspirations. Srdjevic and Srdjevic [24] proposed an AHP-based GDM. Tapia-Rosero et al. [25] proposed a method in the context of GDM. Chen et al. [10] introduced a survey for GDM with heterogeneous preference structures.

Since uncertain and imprecise assessment often occurs in some actual decision processes, a DM may provide his/her preferences over alternatives with imprecision information. In this instance, the linguistic variables $[4,26,27]$ and the intuitionistic fuzzy information [28] might be more suitable for dealing with them.

Zadeh [29] introduced fuzzy set theory. Since its appearance, the fuzzy set theory has received more and more attention and has been used in a wide range of applications. Readers interested in the fuzzy sciences can refer to a general overview of the related research [30]. However, the fuzzy set only assigns a membership degree to each element, and the representation of non-membership is a complement to membership. It actually ignores the DM's hesitation in the decision process. In 1986, Atanassov [28] introduced the concept of intuitionistic fuzzy set, which emerged from the simultaneous consideration of the degrees of membership and non-membership with a degree of hesitancy. Atanassov's intuitionistic fuzzy sets have better flexibility and practicality in the treatment of fuzzy information and uncertainty than ordinary fuzzy sets. Since the socio-economic environment becomes more complex, the preference information is usually imprecise. That is to say, there may be hesitation or uncertainty about preferences because a decision may be made under time pressure and lack of knowledge or data, or the DMs may have limited attention and information processing capabilities. In such cases, it is suitable and convenient to express the DMs' preferences in an Intuitionistic Fuzzy Number (IFN) [31].

The intuitionistic fuzzy theory has attracted scholars' attention more and more [32-34]. For example, Das and Kar [35] proposed an intuitionistic fuzzy soft set approach to GDM medical system. Hashemi et al. [36] offered a compromise ratio method with an application to water resources management. Hashemi et al. [37] suggested an extended compromise ratio model with an application to reservoir flood control operation under an interval-valued intuitionistic fuzzy environment. Vahdani et al. [14] introduced a design for the elimination and choice translating reality method for GDM in an intuitionistic fuzzy environment. Mousavi et al. [38] presented a model of intuitionistic fuzzy VIKOR in GDM problems. Mousavi et al. [39] developed an intuitionistic fuzzy grey model for selection problems with an application to the inspection planning in manufacturing firms. Liu [40] introduced a GDM method based on intuitionistic linguistic power generalized aggregation operators. Hashemi et al. [37] modeled an extended compromise ratio with an application to reservoir flood control operation under an interval-valued intuitionistic fuzzy environment. 'Intepe et al. [41] modeled a forecasting method with intuitionistic fuzzy information.

A key procedure in the GDM problems is to aggregate all individual decisions into a collective decision. There are numerous aggregation methods, in which the weighted average is a common method and how to determine the weight of DM is one of the key techniques. Yue and Jia [42] proposed a TOPSIS (Technique for Order Preference by Similarity to Ideal Solution) method to determine the weights of DMs. Meng et al. [43] utilized a distance measure to derive the weights of DMs. Wan and Dong [44] utilized a similarity degree to determine the DMs' weights. Zhang and $\mathrm{Xu}$ [45] constructed a goal-programming model to derive the weights of DMs. Wan et al. [46] introduced a method for determining the DMs' weights based on the similarity and proximity degrees.

As we all know, an alternative with respect to multiple attributes can be characterized by an attribute vector or by an attribute matrix. It is common that the separations between attribute vectors/matrices and their ideal solutions/decisions are measured by the Euclidean distance or by the Hamming distance. However, it is not enough because their included angles are ignored. To simultaneously consider the distance measure and the included angle between two decision objects, the objective of this research is to establish a new GDM method in which the weights of DMs are determined by using projection-based method [47]. The technical procedure includes the following steps:

1. An ideal decision is established, which is an average of all individual decisions;

2. The projection of each individual decision on its ideal decision is measured;

3. The weight of each DM, based on its individual importance and an optimal coefficient, is determined by using its projection measurement;

4. All individual decisions are aggregated into a col- 
lective decision by using the weighted averaging operator;

5. Based on the collective decision, the preference order of alternatives is made by using the score and accuracy functions of the IFNs.

Compared with the Euclidean distance or the Hamming distance, projection measurement is a comprehensive consideration. It has been widely used in many decision making problems. For example, using projection measure instead of distance measure, Yue and Jia [48] introduced a GDM model with hybrid intuitionistic fuzzy information. $\mathrm{Xu}$ and $\mathrm{Hu}$ [49] established two projection models for GDM problems. Wei [50] proposed an MADM method based on the projection technique, in which the attribute values were characterized by IFNs. Zheng et al. [51] developed an application of improved grey projection method. Yue [52] proposed a GDM method based on the projection measurement. $\mathrm{Xu}$ and Liu [53] described a GDM approach based on a projection method in uncertain fuzzy environment. Xu and Da [54] modeled an uncertain MADM method. Yue [55] suggested a GDM method based on a projection method. Yue and Jia [56] proposed a direct projection-based GDM methodology with crisp values and interval data.

The motivation and contribution of this paper are:

1. This work is intended to provide a novel GDM approach with aggregation based on the weighted average of all individual decisions;

2. The weight of DM is based on a uniform importance of DM, an individual importance of DM, and an optimal coefficient;

3. The study is aimed to develop a high-resolution decision method in an intuitionistic fuzzy environment.

To introduce our work, the rest of this paper is organized as follows. Section 2 introduces some related concepts. Section 3 describes the proposed method in detail. Section 4 gives a comparison with a related GDM method. Section 5 illustrates the proposed method by using a numerical example and an experimental analysis. Conclusions and further research are drawn in Section 6.

\section{Preliminaries}

As a preparation for introducing our work, some related concepts are illustrated in this section.

Zadeh [29] gave the concept of fuzzy set. As a generalization of Zadeh's fuzzy set, Atanassov [28] introduced the intuitionistic fuzzy set as follows.

Definition 1: Let $X$ be a fixed set; an intuitionistic fuzzy set $A$ in $X$ is defined as $A=\left\{<x, \mu_{A}(x), \nu_{A}(x)\right\rangle$ $\mid x \in X\}$, which is characterized by a membership function, $\mu_{A}: X \rightarrow[0,1]$, and a non-membership function, $\nu_{A}: X \rightarrow[0,1]$, with the condition $0 \leq$ $\mu_{A}(x)+\nu_{A}(x) \leq 1$, for all $x \in X$, where the numbers $\mu_{A}(x)$ and $\nu_{A}(x)$ represent the membership degree and non-membership degree of the element $x$ to the set $A$ respectively.

For an intuitionistic fuzzy set $A$ in $X$, if $\pi_{A}(x)=$ $1-\mu_{A}(x)-\nu_{A}(x), \forall x \in X$, then $\pi_{A}(x)$ is called the indeterminacy degree [28] or hesitancy degree [57] as to whether $x$ belongs to $A$ or not. The larger $\pi_{A}(x)$, the more indeterminacy degree of the knowledge about $x$. Especially, if $\mu_{A}(x)+\nu_{A}(x)=1$, for all elements in universe, the intuitionistic fuzzy set $A$ is reduced to an ordinary fuzzy set $[58,59]$.

For convenience, $\mathrm{Xu}$ and Cai [31] called:

$$
\alpha=(\mu, \nu)
$$

an IFN, such that $\mu, \nu \in[0,1], \mu+\nu \leq 1$ and $\pi=$ $1-\mu-\nu$.

$\mathrm{Xu}$ and Cai [31] introduced some operations related to IFNs as follows:

Definition 2: Let $\alpha=\left(\mu_{\alpha}, \nu_{\alpha}\right)$ and $\beta=\left(\mu_{\beta}, \nu_{\beta}\right)$ be two IFNs and $\lambda$ be a real number; then:

1. $\alpha+\beta=\left(\mu_{\alpha}+\mu_{\beta}-\mu_{\alpha} \mu_{\beta}, \nu_{\alpha} \nu_{\beta}\right)$;

2. $\lambda \alpha=\left(1-\left(1-\mu_{\alpha}\right)^{\lambda}, \nu_{\alpha}^{\lambda}\right), \lambda>0$.

Definition 3 [60]: $\quad$ Let $\alpha_{j}=\left(\mu_{j}, \nu_{j}\right)(j=1,2, \cdots, n)$ be $n$ IFNs and $w=\left(w_{1}, w_{2}, \cdots, w_{n}\right)$ be the weight vector of $\alpha_{j}$, with $w_{j} \geq 0, \sum_{j=1}^{n} w_{j}=1$; then:

$$
\alpha(w)=\sum_{j=1}^{n} w_{j} \alpha_{j}
$$

is called intuitionistic fuzzy weighted average of $\alpha_{j}(j=$ $1,2, \cdots, n)$.

Based on Definition 2, we rewrite Eq. (2) as:

$$
\alpha(w)=\left(1-\prod_{j=1}^{n}\left(1-\mu_{j}\right)^{w_{j}}, \prod_{j=1}^{n} \nu_{j}^{w_{j}}\right) .
$$

Especially, if $w=(1 / n, 1 / n, \cdots, 1 / n)$, then $\alpha(w)$ is reduced to the arithmetic average of $\alpha_{j}(j=1,2, \cdots, n)$ :

$$
\alpha(w)=\left(1-\prod_{j=1}^{n}\left(1-\mu_{j}\right)^{1 / n}, \prod_{j=1}^{n}\left(\nu_{j}\right)^{1 / n}\right) .
$$

Let $\alpha_{1}=\left(\mu_{1}, \nu_{1}\right), \alpha_{2}=\left(\mu_{2}, \nu_{2}\right), \cdots$, and $\alpha_{n}=\left(\mu_{n}, \nu_{n}\right)$ be $n$ IFNs; combining Eqs. (3) and (4), we can obtain their sum as follows:

$$
\sum_{j=1}^{n} \alpha_{j}=\left(1-\prod_{j=1}^{n}\left(1-\mu_{j}\right), \prod_{j=1}^{n} \nu_{j}\right) .
$$


Definition $4[47,49]$ : Let $\alpha=\left(\mu_{\alpha}, \nu_{\alpha}\right)$ and $\beta=$ $\left(\mu_{\beta}, \nu_{\beta}\right)$ be two IFNs; then:

$$
|\alpha|=\sqrt{\mu_{\alpha}^{2}+\nu_{\alpha}^{2}+\pi_{\alpha}^{2}}
$$

is called the module of $\alpha$, where, by Eq. (1), $\pi_{\alpha}=$ $1-\mu_{\alpha}-\nu_{\alpha} ;$ and:

$$
\alpha \cdot \beta=\mu_{\alpha} \mu_{\beta}+\nu_{\alpha} \nu_{\beta}+\pi_{\alpha} \pi_{\beta},
$$

is called the inner/scalar product between $\alpha$ and $\beta$, where, $\pi_{\alpha}$ is same as that in Eq. (6) and $\pi_{\beta}=1-\mu_{\beta}-$ $\nu_{\beta} ;$ and:

$$
\cos (\alpha, \beta)=\frac{\alpha \cdot \beta}{|\alpha||\beta|},
$$

is called the cosine of the included angle between $\alpha$ and $\beta$, and:

$$
\operatorname{Proj}_{\beta}(\alpha)=\frac{\mu_{\alpha} \mu_{\beta}+\nu_{\alpha} \nu_{\beta}+\pi_{\alpha} \pi_{\beta}}{\sqrt{\mu_{\beta}^{2}+\nu_{\beta}^{2}+\pi_{\beta}^{2}}},
$$

is called the projection of $\alpha$ on $\beta$, where, $\operatorname{Proj}_{\beta}(\alpha)=$ $(\alpha \cdot \beta) /|\beta| ; \pi_{\alpha}$ and $\pi_{\beta}$ are same as those in Eq. (7).

In general, the larger the value of $\operatorname{Proj}_{\beta}(\alpha)$, the closer $\alpha$ is to $\beta$. The projection measurement can be illustrated in Figure 1.

Definition 5: Let $X=\left(x_{i j}\right)_{m \times n}$ be a matrix. If all the elements $x_{i j}$ are IFNs, then $X$ is called an intuitionistic fuzzy matrix.

Similar to the projection between two IFNs, we introduce the projection between two intuitionistic fuzzy matrices below.

Definition 6: Let $X=\left(\left(\mu_{i j}, \nu_{i j}\right)\right)_{m \times n}$ and $Y=$ $\left(\left(\xi_{i j}, \eta_{i j}\right)\right)_{m \times n}$ be two intuitionistic fuzzy matrices; then:

$$
\operatorname{Proj}_{Y}(X)=\frac{\sum_{i=1}^{m} \sum_{j=1}^{n}\left(\mu_{i j} \xi_{i j}+\nu_{i j} \eta_{i j}+\pi_{i j} \rho_{i j}\right)}{\sqrt{\sum_{i=1}^{m} \sum_{j=1}^{n}\left(\left(\xi_{i j}\right)^{2}+\left(\eta_{i j}\right)^{2}+\left(\rho_{i j}\right)^{2}\right)}},
$$

is called the projection of $X$ on $Y$, where, by Eq. (1), $\pi_{i j}=1-\mu_{i j}-\nu_{i j}$, and $\rho_{i j}=1-\xi_{i j}-\eta_{i j}(i=$ $1,2, \cdots, m, j=1,2, \cdots, n)$.

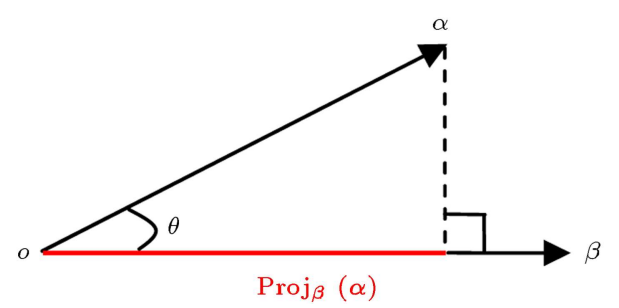

Figure 1. The projection of $\alpha$ on $\beta$.
Chen and Tan [61] introduced a score function $s$ of an IFN as follows.

Let $\alpha=(\mu, \nu)$ be an IFN; the score of $\alpha$ can be evaluated by the score function $s$ shown as:

$$
s(\alpha)=\mu-\nu,
$$

where $s(\alpha) \in[-1,1]$. The larger score $s(\alpha)$ means that the IFN $\alpha$ is greater.

The following example shows that some IFNs cannot be graded by the score function.

Example 1: Let $\alpha=(0.1,0.1)$ and $\beta=(0.5,0.5)$ be two IFNs; by applying Eq. (10), we obtain that $s(\alpha)=$ $s(\beta)=0$. In this case, we do not know which IFN is greater.

Later, Hong and Choi [62] added an accuracy function $h$ as:

$$
h(\alpha)=\mu+\nu,
$$

to evaluate the degree of accuracy of the IFN $\alpha=$ $(\mu, \nu)$, where $h(\alpha) \in[0,1]$. The larger value of $h(\alpha)$ means that $\alpha$ has the greater degree of accuracy.

For $\alpha=(0.1,0.1)$ and $\beta=(0.5,0.5)$ given in Example 1, according to the accuracy function $h$, we have $h(\alpha)=0.2$ and $h(\beta)=1$; thereby, we have $\alpha<\beta$.

Based on the score function $s$ and the accuracy function $h, \mathrm{Xu}$ and Cai [31] gave an order relation between any pair of IFNs, which is defined as follows.

Definition 7: Let $\alpha=\left(\mu_{\alpha}, \nu_{\alpha}\right)$ and $\beta=\left(\mu_{\beta}, \nu_{\beta}\right)$ be two IFNs, and $s$ and $h$ be score function and accuracy function, respectively; then:

1. If $s(\alpha)<s(\beta)$, then $\alpha<\beta$;

2. If $s(\alpha)=s(\beta)$, then:
a) If $h(\alpha)=h(\beta)$, then $\alpha=\beta$;
b) If $h(\alpha)<h(\beta)$, then $\alpha<\beta$.

\section{Projection-based approach to group decision making}

For convenience, throughout this paper, the following index sets and the key elements are used in the decision process.
Index:
$i: \quad$ Index for alternatives, $i \in M=$ $\{1,2, \cdots, m\}$
$j: \quad$ Index for attributes, $j \in N=$ $\{1,2, \cdots, n\}$
$k: \quad$ Index for DMs, $k \in T=\{1,2, \cdots, t\}$. 
Key elements for GDM:

1. A set of $m$ feasible alternatives written as $A=$ $\left\{A_{1}, A_{2}, \cdots, A_{m}\right\}(m \geq 2)$;

2. A set of attributes written as $U=\left\{u_{1}, u_{2}, \cdots, u_{n}\right\}$;

3. A weight vector of attributes written as $w=$ $\left(w_{1}, w_{2}, \cdots, w_{n}\right)$, with $0 \leq w_{j} \leq 1$ and $\sum_{j=1}^{n} w_{j}=$ 1 ;

4. A set of DMs written as $D=\left\{d_{1}, d_{2}, \cdots, d_{t}\right\}$;

5. A weight vector of DMs written as $\lambda=\left(\lambda_{1}\right.$, $\left.\lambda_{2}, \cdots, \lambda_{t}\right)$, with $0 \leq \lambda_{k} \leq 1$ and $\sum_{k=1}^{t} w_{k}=1$.

A GDM problem can be described in the following steps.

\subsection{Determining the weights of decision makers}

First, each DM employs the linguistic variables [27,63] to evaluate the attribute values and the grades of attributes. Let:

$$
X_{k}=\left(x_{i j}^{k}\right)_{m \times n}, \quad h_{k}=\left(h_{1}^{k}, h_{2}^{k}, \cdots, h_{n}^{k}\right), \quad k \in T,
$$

be the decision matrix of the $k$ th DM and the grades of attributes, in which the decision information, $x_{i j}^{k}$ and $h_{j}^{k}$, are expressed by linguistic variables.

For convenience, we convert the linguistic variables in $X_{k}$ into the IFNs and the grades $h_{k}$ into their weights, shown as:

$$
Y_{k}=\left(y_{i j}^{k}\right)_{m \times n}, \quad w_{k}=\left(w_{1}^{k}, w_{2}^{k}, \cdots, w_{n}^{k}\right), \quad k \in T,
$$

where $y_{i j}^{k}=\left(\mu_{i j}^{k}, \nu_{i j}^{k}\right)(i \in M, j \in N, k \in T)$ are IFNs. $w_{j}^{k}(j \in N, k \in T)$ are the weights of attributes, with $0 \leq w_{j}^{k} \leq 1$ and $\sum_{j=1}^{n} w_{j}^{k}=1$.

In the next step, we assign each weight, $w_{j}^{k}(j \in$ $N)$, to its corresponding attribute, $u_{j}$; then the weighted decision matrix is obtained as follows:

$$
R_{k}=\left(r_{i j}^{k}\right)_{m \times n}, \quad k \in T,
$$

where, $r_{i j}^{k}=w_{j}^{k} y_{i j}^{k}=w_{j}^{k}\left(\mu_{i j}^{k}, \nu_{i j}^{k}\right)=\left(\tau_{i j}^{k}, v_{i j}^{k}\right)$; by Definition $2, \tau_{i j}^{k}=1-\left(1-\mu_{i j}^{k}\right)^{w_{j}^{k}}$ and $v_{i j}^{k}=\left(\nu_{i j}^{k}\right)^{w_{j}^{k}}(i \in$ $M, j \in N, k \in T)$.

Then, we hope to find an ideal decision among all weighted decisions, $R_{k}(k \in T)$. Inspired by the idea in literature [64], the average of all individual decisions in Eq. (14) should be an ideal decision as follows:

$$
R_{*}=\left(r_{i j}^{*}\right)_{m \times n},
$$

where, $r_{i j}^{*}=\left(\tau_{i j}^{*}, v_{i j}^{*}\right)$ and by Eq. (4), $\tau_{i j}^{*}=1-$ $\prod_{k=1}^{t}\left(1-\tau_{i j}^{k}\right)^{1 / t}$ and $v_{i j}^{*}=\prod_{k=1}^{t}\left(v_{i j}^{k}\right)^{1 / t}(i \in M, j \in$ $N)$.
The following work is to determine the weights of DMs. The basic idea is very straightforward, that is, the larger the projection of $R_{k}$ on $R_{*}$, the better the decision $R_{k}$ is and the larger the weight of the $k$ th DM is.

According to Eq. (9), the projection of $R_{k}$ on $R_{*}$ can be calculated by:

$$
\operatorname{Proj}_{R_{*}}\left(R_{k}\right)=\frac{\sum_{i=1}^{m} \sum_{j=1}^{n}\left(\tau_{i j}^{k} \tau_{i j}^{*}+v_{i j}^{k} v_{i j}^{*}+\pi_{i j}^{k} \pi_{i j}^{*}\right)}{\sqrt{\left.\sum_{i=1}^{m} \sum_{j=1}^{n}\left(\tau_{i j}^{*}\right)^{2}+\left(v_{i j}^{*}\right)^{2}+\left(\pi_{i j}^{*}\right)^{2}\right)}},
$$

$k \in T$,

where, by Eq. (1), $\pi_{i j}^{k}=1-\tau_{i j}^{k}-v_{i j}^{k}$ and $\pi_{i j}^{*}=1-$ $\tau_{i j}^{*}-v_{i j}^{*}(i \in M, j \in N, k \in T)$.

Thus, a projection-based closeness coefficient is derived by Eq. (16) as follows:

$$
C_{k}=\frac{\operatorname{Proj}_{R_{*}}\left(R_{k}\right)}{\sum_{k=1}^{t} \operatorname{Proj}_{R_{*}}\left(R_{k}\right)}, \quad k \in T
$$

such that $0 \leq C_{k} \leq 1$.

$C_{k}$ is a measure of importance of the $k$ th DM. It can measure the degree of closeness that the $k$ th DM's opinion approaches the average of the group's opinions. We call it a uniform importance.

Furthermore, in many cases, a DM is an expert in an area (the main reason of GDM) and his/her opinion in that area may be much more important than the opinions of the others. This relative importance can also be measured. For example, a member of the Chinese Academy of Engineering/Sciences (at a national level), a famous expert in a province (at a provincial level), and a famous expert in a local city (at a local level), in China can be scored 1, 0.8, and 0.6 , respectively. The importance of an expert in his/her area is called the individual importance in this paper, which is written as $I_{k}$. To keep the measure $I_{k}$ consistent with $C_{k}$, we restrict it to $0 \leq I_{k} \leq 1$.

Based on the above-mentioned reasons, the weight $\lambda_{k}$ of DM is determined by:

$$
\lambda_{k}=\alpha C_{k}+(1-\alpha) I_{k}, \quad k \in T,
$$

where $\alpha(0 \leq \alpha \leq 1)$ is an optimal coefficient and $\lambda_{k}$ satisfies $\lambda_{k} \geq 0$ and $\sum_{k=1}^{t} \lambda_{k}=1$.

\subsection{Preference ranking of alternatives}

For the weight vector, $\lambda=\left(\lambda_{1}, \lambda_{2}, \cdots, \lambda_{t}\right)$, of DMs, we aggregate all individual decisions, $R_{k}$, in Eq. (14) into a collective decision as follows:

$$
R=\left(r_{i j}\right)_{m \times n},
$$


where:

$$
\begin{aligned}
R & =\sum_{k=1}^{t} \lambda_{k} R_{k}, r_{i j}=\sum_{k=1}^{t} \lambda_{k} r_{i j}^{k}=\sum_{k=1}^{t} \lambda_{k}\left(\tau_{i j}^{k}, v_{i j}^{k}\right) \\
& =\left(\iota_{i j}, \kappa_{i j}\right)
\end{aligned}
$$

and by Eq. (3), $\iota_{i j}=1-\prod_{k=1}^{t}\left(1-\tau_{i j}^{k}\right)^{\lambda_{k}}$ and $\kappa_{i j}=$ $\prod_{k=1}^{t}\left(v_{i j}^{k}\right)^{\lambda_{k}}(i \in M, j \in N)$.

In the following sections, we will focus on the ranking of alternatives, which is also the aim of GDM. Summing all elements in each line of $R$ in Eq. (19), we have the overall attribute value of each alternative as follows:

$$
\alpha_{i}=\left(\iota_{i}, \kappa_{i}\right), i \in M
$$

where, by Eq. (5), $\left(\iota_{i}, \kappa_{i}\right)=\sum_{j=1}^{n} r_{i j}=\left(1-\prod_{j=1}^{n}(1-\right.$ $\left.\left.\iota_{i j}\right), \prod_{j=1}^{n} \kappa_{i j}\right)$.

We can utilize Eqs. (11) and (12) to calculate the score and accuracy degree of the overall value $\alpha_{i}$ in Eq. (20), respectively. After that, we rank the alternatives according to Definition 7 .

\subsection{Procedure of the proposed model}

In sum, an algorithm for GDM, based on the projection measurement, is given in the following steps:

- Step 1. Determine individual decision information. Each DM $d_{k}$ provides his/her decision matrix $X_{k}=\left(x_{i j}^{k}\right)_{m \times n}$, on alternatives with respect to attributes and grade vector, $h_{k}=\left(h_{1}^{k}, h_{2}^{k}, \cdots, h_{n}^{k}\right)$, of attributes, which are shown in Eq. (12);

- Step 2. Convert the decision information. The decision information in $X_{k}$, expressed by linguistic variables, can be converted to IFNs and the attributes' grades in $h_{k}$, expressed by linguistic variables, can be converted to their weights expressed by non-negative real numbers, which are shown in Eq. (13);

- Step 3. Construct the weighted individual decision. For the attributes' weight vector $\left(w_{1}^{k}, w_{2}^{k}, \cdots, w_{n}^{k}\right)$ given by the $k$ th $\mathrm{DM}$, the weighted individual decision matrix $R_{k}$ is constructed by Eq. (14);

- Step 4. Determine the ideal decision of all individual decisions. The ideal decision of all individual decisions, $R_{k}(k \in T), R_{*}$, is determined by Eq. (15);

- Step 5. Calculate the projection of each individual decision on the ideal decision. The projection of each individual decision, $R_{k}$, on the ideal decision, $R_{*}$, $\operatorname{Proj}_{R_{*}}\left(R_{k}\right)$, is calculated by Eq. (16);

- Step 6. Calculate the uniform importance of DM. A projection-based uniform importance of the $k$ th $\mathrm{DM}, C_{k}$, is calculated by Eq. (17);
- Step 7. Calculate the weights of DMs. For the individual importance $I_{k}$ and the uniform importance $C_{k}$, the weights of DMs can be calculated by Eq. (18);

- Step 8. Calculate the collective decision. The collective decision $R$ of the group can be obtained by Eq. (19);

- Step 9. Calculate the overall attribute value for each alternative. The overall attribute value of alternative $A_{i}, \alpha_{i}(i \in M)$, can be calculated by Eq. (20);

- Step 10. Calculate the score and accuracy degree of the overall attribute value of each alternative. The score and accuracy degree of the overall attribute values of alternative $A_{i}, s\left(\alpha_{i}\right)$, and $h\left(\alpha_{i}\right)(i \in M)$ are calculated by Eqs. (10) and (11), respectively;

- Step 11. Rank the preference order of alternatives. According to Definition 7, all the alternatives are ranked in accordance with their scores and accuracy degrees of the overall attribute values.

To realize this algorithm, a framework is illustrated in Figure 2.

\section{Comparison with another method}

In this section, we compare the proposed approach with another projection-based GDM method in an intuitionistic fuzzy environment.

Yue [47] introduced a GDM approach, in which the alternatives were directly ranked based on an extended TOPSIS technique. It is related to the current approach. There are two contributions in literature [47]:

1. The separation measure in the TOPSIS technique is replaced by a projection measurement;

2. An optimistic coefficient of relative closeness is established [47].

However, the DM's importance is not taken into consideration. In fact, in many cases, the importance of the different DMs may be different. The differences of this work from the literature [47] are as follows.

First, the weights of DMs are the same in model [47], which does not consider the difference of DM's importance; whereas the current GDM method pays much attention to the weights of DMs and provides a novel projection-based method to implement it. An individual importance $I_{k}$ is added to the comprehensive relative coefficient in order to determine the comprehensive weighs of DMs.

Second, the alternatives are ranked by the TOPSIS technique in the literature [47], in which some negative ideal decisions are established; however, the current model has no negative ideal decision and the 


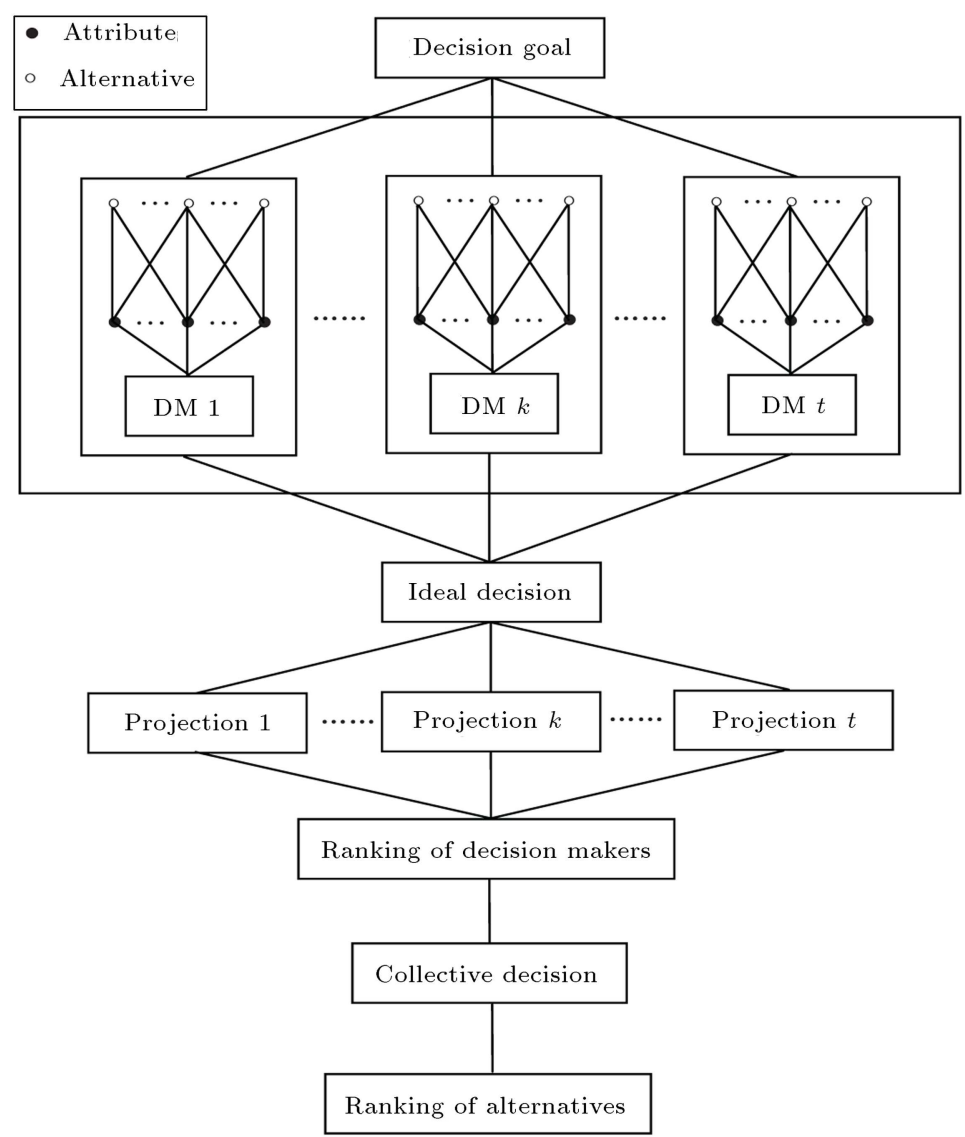

Figure 2. Framework of the projection-based approach to group decision making.

alternatives are ranked only through an (positive) ideal decision (an average of all individual decisions). Thus, the current model is simpler than the model in [47].

Finally, there are no aggregations of individual decisions in model [47]. All alternatives are ranked directly based on their relative closeness to an optimistic coefficient; on the contrary, our method in this paper aggregates all individual decisions into a collective decision. All alternatives are ranked based on their scores and accuracies. In a sense, the current method is simple and the two methods are complementary.

\section{Experimental analysis}

\subsection{Illustrative example}

To test the contribution of our approach, a real-life supplier evaluation and selection problem is solved by the proposed projection-based approach in the following.

An automotive manufacturing company desires to select a suitable material supplier to purchase a key component of automobile part manufacturing. To evaluate and select the suitable supplier(s), a committee, including three DMs in the company, is set up; the DMs are:

$d_{1} \quad$ The production manager

$d_{2} \quad$ The quality manger $d_{3} \quad$ The planning manager

Four attributes considered here are as follows:

$u_{1} \quad$ Product quality

$u_{2} \quad$ Technical specification

$u_{3} \quad$ Delivery performance

$u_{4} \quad$ flexibility

After the preliminary screening, three candidates as alternatives remain for the latter evaluation, which are written as $\left\{A_{1}, A_{2}, A_{3}\right\}$. For the performance grades of attributes, the linguistic terms [27] and their corresponding scores are shown in Table 1.

Based on Table 1, the linguistic variables can be converted into their scores; then, the weights

Table 1. Linguistic terms and corresponding scores for rating the grades of attributes.

\begin{tabular}{lc}
\hline \multicolumn{1}{c}{ Linguistic variable } & Score \\
\hline Very Unimportant (VU) & 1 \\
Unimportant (U) & 2 \\
Medium (M) & 3 \\
Important (I) & 4 \\
Very Important (VI) & 5 \\
\hline
\end{tabular}


of attributes are obtained. A specific conversion is introduced in the following example.

Example 2: Let $\left(h_{1}, h_{2}, h_{3}, h_{4}\right)=(\mathrm{VI}, \mathrm{M}, \mathrm{M}, \mathrm{VI})$ be the grades of attributes $\left\{u_{1}, u_{2}, u_{3}, u_{4}\right\}$, which are given by a DM. According to Table 1, we first convert the linguistic variables (VI, M, M, VI) into the scores $(5,3,3,5)$. Then, we need to normalize the scores $(5,3,3,5)$ as $\left(w_{1}, w_{2}, w_{3}, w_{4}\right)$, where $w_{1}=5 /$ sum $=$ $0.3125, w_{2}=3 / \mathrm{sum}=0.1875, w_{3}=3 / \mathrm{sum}=0.1875$, $w_{4}=5 /$ sum $=0.3125$, and sum $=5+3+3+5=16$.

Table 2 introduces the linguistic terms for rating the attribute values and corresponding IFNs, which are also described by the 5 -point scales.

Three DMs employ the linguistic variables with the 5-point scales to assess the candidates with respect to attributes and the grades of attributes, which are shown in Table 3.

According to Step 2, the potential suppliers' grades in Table 3, expressed by linguistic variables, can be converted to IFNs, and the attributes' grades

Table 2. Linguistic terms and corresponding IFNs for rating attribute values.

\begin{tabular}{lc}
\hline \multicolumn{1}{c}{ Linguistic variable } & IFN \\
\hline Strong Dissatisfaction (SD) & $(0.05,0.90)$ \\
Dissatisfaction (D) & $(0.20,0.70)$ \\
Fair (F) & $(0.50,0.30)$ \\
Satisfaction (S) & $(0.70,0.20)$ \\
Strong Satisfaction (SS) & $(0.90,0.05)$ \\
\hline
\end{tabular}

Table 3. Candidates' evaluation and attributes' grades in linguistic variables.

\begin{tabular}{cccccc}
\hline DM & $\begin{array}{c}\text { Candidate and } \\
\text { grade of attribute }\end{array}$ & $\boldsymbol{u}_{\mathbf{1}}$ & $\boldsymbol{u}_{\mathbf{2}}$ & $\boldsymbol{u}_{\mathbf{3}}$ & $\boldsymbol{u}_{4}$ \\
\hline$d_{1}$ & $A_{1}$ & $\mathrm{~S}$ & $\mathrm{D}$ & $\mathrm{F}$ & $\mathrm{F}$ \\
& $A_{2}$ & $\mathrm{SS}$ & $\mathrm{SD}$ & $\mathrm{S}$ & $\mathrm{S}$ \\
& $A_{3}$ & $\mathrm{~S}$ & $\mathrm{D}$ & $\mathrm{S}$ & $\mathrm{SD}$ \\
& $h_{1}$ & $\mathrm{VI}$ & $\mathrm{M}$ & $\mathrm{M}$ & $\mathrm{VI}$ \\
& & & & & \\
$d_{2}$ & $A_{1}$ & $\mathrm{~S}$ & $\mathrm{~F}$ & $\mathrm{D}$ & $\mathrm{S}$ \\
& $A_{2}$ & $\mathrm{~F}$ & $\mathrm{~S}$ & $\mathrm{D}$ & $\mathrm{F}$ \\
& $A_{3}$ & $\mathrm{~F}$ & $\mathrm{~S}$ & $\mathrm{SS}$ & $\mathrm{S}$ \\
& $h_{2}$ & $\mathrm{I}$ & $\mathrm{VI}$ & $\mathrm{M}$ & $\mathrm{VI}$ \\
& & & & & \\
& $A_{1}$ & $\mathrm{SS}$ & $\mathrm{D}$ & $\mathrm{S}$ & $\mathrm{SS}$ \\
$d_{3}$ & $A_{2}$ & $\mathrm{SS}$ & $\mathrm{D}$ & $\mathrm{S}$ & $\mathrm{S}$ \\
& $A_{3}$ & $\mathrm{~F}$ & $\mathrm{SD}$ & $\mathrm{SS}$ & $\mathrm{S}$ \\
& $h_{3}$ & $\mathrm{M}$ & $\mathrm{I}$ & $\mathrm{M}$ & $\mathrm{M}$ \\
\hline
\end{tabular}

in Table 3, expressed by linguistic variables, can be converted to their weights, which are shown in Table 4. For the weights of attributes in Table 4, by Step 3, we can calculate the three weighted individual decisions, which are summarized in Table 5 .

The ideal decision of all individual decisions is determined by Step 4, which is shown in Table 6 .

The projection of each individual decision, $R_{k}$, on the ideal decision, $R_{*}$, is calculated by Step 5 . The uniform importance of DM is calculated by Step 6 . The individual weights of three DMs, $\left(I_{1}, I_{2}, I_{3}\right)=$ $(0.4,0.3,0.3)$, are given according to the social influence of each DM as an expert. The optimistic coefficient $\alpha=$ 0.6 is given by all DMs. Also, the weights $\lambda_{k}$ of DMs are calculated by Step 7, which are summarized in Table 7 .

For the weight vector $\left(\lambda_{1}, \lambda_{2}, \lambda_{3}\right)=(0.3681$, $0.3139,0.3180)$ of $\mathrm{DMs}$, we can calculate the collective decision of the group by Step 8, which is shown in Table 8.

The overall attribute value of each candidate is calculated by Step 9 ; the score of each candidate is calculated by Step 10; and all candidates are ranked by Step 11, which are summarized in Table 9 .

Table 9 shows that the ranking of candidates:

$$
A_{2} \succ A_{1} \succ A_{3},
$$

where $A \succ B$ represents that $A$ is superior to $B$. That is to say, $A_{2}$ is to look for the best or the most suitable supplier among the three potential suppliers in this automotive manufacturing company, followed by $A_{1}$ and $A_{3}$.

\subsection{Sensitivity analysis for the optimistic coefficient}

The optimistic coefficient $\alpha$ in Eq. (18) is very important, which should be determined before Eq. (18) is used. To show feasibility and effectiveness in Eq. (21), we would like to know how $\alpha$ impacts the overall ranking of the candidates. To intuitively illustrate the relation between the rankings of potential suppliers as $\alpha$ increases from 0 to 1 , we geometrically show it in Figure 3.

If $\alpha=0$, then the scores of three candidates $A_{1}$, $A_{2}$, and $A_{3}$ are $0.3850,0.4508$, and 0.3383 , respectively. In this case, $A_{2}$ is the best candidate, followed by $A_{1}$ and $A_{3}$. When $\alpha$ increases from 0 to 1 , Figure 3 shows that the rankings of three potential suppliers do not change, and the difference between their grades is obvious. This outcome shows that Eq. (18) is robust and it provides the same order for different values of $\alpha$.

\subsection{Experimental analysis of different distance measures}

In this subsection, we compare our result in Table 9 with different distance measures: the Euclidean distance and the Hamming distance. 
Table 4. Candidates' evaluation in IFNs and weights of attributes.

\begin{tabular}{cccccc}
\hline DM & $\begin{array}{c}\text { Candidate and } \\
\text { weight of attribute }\end{array}$ & $\boldsymbol{u}_{\mathbf{1}}$ & $\boldsymbol{u}_{\mathbf{2}}$ & $\boldsymbol{u}_{\mathbf{3}}$ & $\boldsymbol{u}_{\mathbf{4}}$ \\
\hline \multirow{4}{*}{$d_{1}$} & $A_{1}$ & $(0.70,0.20)$ & $(0.20,0.70)$ & $(0.50,0.30)$ & $(0.50,0.30)$ \\
& $A_{2}$ & $(0.90,0.05)$ & $(0.05,0.90)$ & $(0.70,0.20)$ & $(0.70,0.20)$ \\
& $A_{3}$ & $(0.70,0.20)$ & $(0.20,0.70)$ & $(0.70,0.20)$ & $(0.05,0.90)$ \\
& $w_{1}$ & 0.3125 & 0.1875 & 0.1875 & 0.3125 \\
& & & & & \\
$d_{2}$ & $A_{1}$ & $(0.70,0.20)$ & $(0.50,0.30)$ & $(0.20,0.70)$ & $(0.70,0.20)$ \\
& $A_{2}$ & $(0.50,0.30)$ & $(0.70,0.20)$ & $(0.20,0.70)$ & $(0.50,0.30)$ \\
& $A_{3}$ & $(0.50,0.30)$ & $(0.70,0.20)$ & $(0.90,0.05)$ & $(0.70,0.20)$ \\
& $w_{2}$ & 0.2353 & 0.2941 & 0.1765 & 0.2941 \\
& & & & & \\
$d_{3}$ & $A_{1}$ & $(0.90,0.05)$ & $(0.20,0.70)$ & $(0.70,0.20)$ & $(0.90,0.05)$ \\
& $A_{2}$ & $(0.90,0.05)$ & $(0.20,0.70)$ & $(0.70,0.20)$ & $(0.70,0.20)$ \\
& $A_{3}$ & $(0.50,0.30)$ & $(0.05,0.90)$ & $(0.90,0.05)$ & $(0.70,0.20)$ \\
& $w_{3}$ & 0.2308 & 0.3076 & 0.2308 & 0.2308 \\
\hline
\end{tabular}

Table 5. Three weighted individual decisions with intuitionistic fuzzy information.

\begin{tabular}{cccccc}
\hline Decision & Candidate & $\boldsymbol{u}_{\boldsymbol{1}}$ & $\boldsymbol{u}_{\mathbf{2}}$ & $\boldsymbol{u}_{\mathbf{3}}$ & $\boldsymbol{u}_{\mathbf{4}}$ \\
\hline \multirow{3}{*}{$R_{1}$} & $A_{1}$ & $(0.3136,0.6047)$ & $(0.0410,0.9353)$ & $(0.1219,0.7979)$ & $(0.1948,0.6864)$ \\
& $A_{2}$ & $(0.5130,0.3921)$ & $(0.0096,0.9804)$ & $(0.2021,0.7395)$ & $(0.3136,0.6047)$ \\
& $A_{3}$ & $(0.3136,0.6047)$ & $(0.0410,0.9353)$ & $(0.2021,0.7395)$ & $(0.0159,0.9676)$ \\
& & & & & \\
$R_{2}$ & $A_{1}$ & $(0.2467,0.6848)$ & $(0.1844,0.7018)$ & $(0.0386,0.9390)$ & $(0.2982,0.6229)$ \\
& $A_{2}$ & $(0.1505,0.7533)$ & $(0.2982,0.6229)$ & $(0.0386,0.9390)$ & $(0.1844,0.7018)$ \\
& $A_{3}$ & $(0.1505,0.7533)$ & $(0.2982,0.6229)$ & $(0.3340,0.5893)$ & $(0.2982,0.6229)$ \\
& & & & & \\
$R_{3}$ & $A_{1}$ & $(0.4122,0.5009)$ & $(0.0663,0.8961)$ & $(0.2426,0.6897)$ & $(0.4122,0.5009)$ \\
& $A_{2}$ & $(0.4122,0.5009)$ & $(0.0663,0.8961)$ & $(0.2426,0.6897)$ & $(0.2426,0.6897)$ \\
& $A_{3}$ & $(0.1478,0.7574)$ & $(0.0157,0.9681)$ & $(0.4122,0.5009)$ & $(0.2426,0.6897)$ \\
\hline
\end{tabular}

Table 6. The ideal decision among all individual decisions.

\begin{tabular}{ccccc}
\hline Candidate & $\boldsymbol{u}_{\boldsymbol{1}}$ & $\boldsymbol{u}_{\mathbf{2}}$ & $\boldsymbol{u}_{\mathbf{3}}$ & $\boldsymbol{u}_{\mathbf{4}}$ \\
\hline$A_{1}$ & $(0.3277,0.5919)$ & $(0.0995,0.8379)$ & $(0.1385,0.8025)$ & $(0.3075,0.5983)$ \\
$A_{2}$ & $(0.3758,0.5289)$ & $(0.1342,0.8180)$ & $(0.1656,0.7824)$ & $(0.2487,0.6640)$ \\
$A_{3}$ & $(0.2079,0.7014)$ & $(0.1282,0.8262)$ & $(0.3215,0.6021)$ & $(0.1943,0.7463)$ \\
\hline
\end{tabular}

Table 7. Projections, uniform importance, individual importance, weights of DMs, and their ranking.

\begin{tabular}{cccccc}
\hline DM & Proj $_{\boldsymbol{R}_{*}}\left(\boldsymbol{R}_{\boldsymbol{k}}\right)$ & $\boldsymbol{C}_{\boldsymbol{k}}$ & $\boldsymbol{I}_{\boldsymbol{k}}$ & $\boldsymbol{\lambda}_{\boldsymbol{k}}$ & Ranking \\
\hline$d_{1}$ & 2.7541 & 0.3468 & 0.4000 & 0.3681 & 1 \\
$d_{2}$ & 2.5665 & 0.3232 & 0.3000 & 0.3139 & 3 \\
$d_{3}$ & 2.6214 & 0.3301 & 0.3000 & 0.3180 & 2 \\
\hline
\end{tabular}

Table 8. Collective decision of the group.

\begin{tabular}{ccccc}
\hline Candidate & $\boldsymbol{u}_{\boldsymbol{1}}$ & $\boldsymbol{u}_{\boldsymbol{2}}$ & $\boldsymbol{u}_{\mathbf{3}}$ & $\boldsymbol{u}_{\mathbf{4}}$ \\
\hline$A_{1}$ & $(0.3273,0.5922)$ & $(0.0963,0.8431)$ & $(0.1381,0.8017)$ & $(0.3022,0.6023)$ \\
$A_{2}$ & $(0.3843,0.5203)$ & $(0.1276,0.8263)$ & $(0.1679,0.7796)$ & $(0.2524,0.6607)$ \\
$A_{3}$ & $(0.2138,0.6960)$ & $(0.1233,0.8324)$ & $(0.3159,0.6084)$ & $(0.1857,0.7567)$ \\
\hline
\end{tabular}


Table 9. Overall attribute values $\alpha_{i}$, scores $s\left(\alpha_{i}\right)$, and the ranking of candidates.

\begin{tabular}{cccc}
\hline Candidate & $\boldsymbol{\alpha}_{\boldsymbol{i}}$ & $\boldsymbol{s}\left(\boldsymbol{\alpha}_{\boldsymbol{i}}\right)$ & Ranking \\
\hline$A_{1}$ & $(0.6344,0.2411)$ & 0.3932 & 2 \\
$A_{2}$ & $(0.6659,0.2215)$ & 0.4444 & 1 \\
$A_{3}$ & $(0.6160,0.2667)$ & 0.3494 & 3 \\
\hline
\end{tabular}

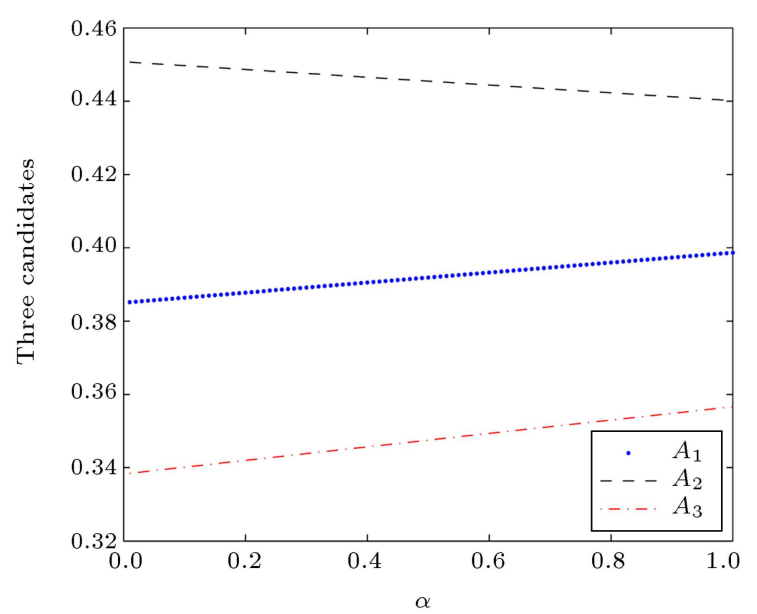

Figure 3. The rankings of three potential suppliers vs. $\alpha$.

If we use the Euclidean distance to replace the projection measurement in the procedure of the proposed model, Eq. (16) will be replaced by:

$s_{E_{k}}=\sqrt{\sum_{i=1}^{m} \sum_{j=1}^{n}\left(\left(\tau_{i j}^{k}-\tau_{i j}^{*}\right)^{2}+\left(v_{i j}^{k}-v_{i j}^{*}\right)^{2}+\left(\pi_{i j}^{k}-\pi_{i j}^{*}\right)^{2}\right)}$,

$k \in T$

where, $s_{E_{k}}=s_{E}\left(R_{k}, R_{*}\right) ; \pi_{i j}^{k}$ and $\pi_{i j}^{*}$ are same as those in Eq. (16). Eq. (17) is replaced by:

$$
C_{k}=1-\frac{s_{E_{k}}}{\sum_{k=1}^{t} s_{E_{k}}}, \quad k \in T
$$

The separation of each $R_{k}(k=1,2,3)$ from the ideal decision $R_{*}$, the uniform importance calculated by Eq. (22), the individual importance, the comprehensive closeness coefficient, the weights of DMs, and their ranking based on the Euclidean distance are shown in Table 10 .

Table 10. Separations, uniform importance, individual importance, weights of DMs, and their ranking based on the Euclidean distance.

\begin{tabular}{cccccc}
\hline $\mathbf{D M}$ & $\boldsymbol{s}_{\boldsymbol{E}_{\boldsymbol{k}}}$ & $\boldsymbol{C}_{\boldsymbol{k}}$ & $\boldsymbol{I}_{\boldsymbol{k}}$ & $\boldsymbol{\lambda}_{\boldsymbol{k}}$ & Ranking \\
\hline$d_{1}$ & 0.5339 & 0.3425 & 0.4000 & 0.3655 & 1 \\
$d_{2}$ & 0.6272 & 0.4023 & 0.3000 & 0.3614 & 2 \\
$d_{3}$ & 0.3978 & 0.2552 & 0.3000 & 0.2731 & 3 \\
\hline
\end{tabular}

If we use the Hamming distance to replace the projection measurement in the procedure of the proposed model, Eq. (16) will be replaced by:

$$
s_{H_{k}}=\sum_{i=1}^{m} \sum_{j=1}^{n}\left(\left|\tau_{i j}^{k}-\tau_{i j}^{*}\right|+\left|v_{i j}^{k}-v_{i j}^{*}\right|+\left|\pi_{i j}^{k}-\pi_{i j}^{*}\right|\right),
$$

$$
k \in T
$$

where, $s_{H_{k}}=s_{H}\left(R_{k}, R_{*}\right)$, and $\pi_{i j}^{k}$ and $\pi_{i j}^{*}$ are same as those in Eq. (17). In this case, Eq. (17) is replaced by:

$$
C_{k}=1-\frac{s_{H_{k}}}{\sum_{k=1}^{t} s_{H_{k}}}, \quad k \in T
$$

The separation of each $R_{k}(k=1,2,3)$ from the ideal decision $R_{*}$, the uniform importance calculated by Eq. (23), the individual importance, the weights of DMs, and their ranking based on the Hamming distance are shown in Table 11.

The scores of three candidates $A_{1}, A_{2}$, and $A_{3}$ with three measures are shown in Table 12. In Table 12, $s_{P}\left(\alpha_{i}\right), s_{E}\left(\alpha_{i}\right)$, and $s_{H}\left(\alpha_{i}\right)$, respectively, represent the scores measured by using the projection measure, the Euclidean distance, and the Hamming distance. Table 12 shows that the rankings based on three different measures are the same. In this case, we do not know which measure is better.

To show the advantage of the current method, we compare three measures based on a resolution of scores of candidates. Specifically, we compare the resolutions of the rankings derived from the measures, and these resolutions are based on their ratios. For example, to show the resolution of ranking $0.4444 \succ$ $0.3932 \succ 0.3494$ based on projection measurement, it can be calculated by two ratios: large/medium $=s_{P}\left(\alpha_{2}\right) / s_{P}\left(\alpha_{1}\right)=0.4444 / 0.3932=1.1302$ and medium $/$ small $=s_{P}\left(\alpha_{1}\right) / s_{P}\left(\alpha_{3}\right)=0.3932 / 0.3494=$ 1.1254. The overall resolution is calculated by $s_{P}\left(\alpha_{2}\right) / s_{P}\left(\alpha_{1}\right)+s_{P}\left(\alpha_{1}\right) / s_{P}\left(\alpha_{3}\right)=1.1302+1.1254=$ 2.2556. Our idea is that the larger the overall resolution a result provides for the scores of candidates, the smaller sensitivity and the higher robustness this measure will have. Therefore, this result is more reliable. The resolutions of rankings based on three measures are shown in Table 13.

Table 11. Separations, uniform importance, individual importance, weights of DMs, and their ranking based on the Hamming distance.

\begin{tabular}{cccccc}
\hline $\mathbf{D M}$ & $\boldsymbol{s}_{\boldsymbol{H}_{\boldsymbol{k}}}$ & $\boldsymbol{C}_{\boldsymbol{k}}$ & $\boldsymbol{I}_{\boldsymbol{k}}$ & $\boldsymbol{\lambda}_{\boldsymbol{k}}$ & Ranking \\
\hline$d_{1}$ & 2.4523 & 0.3393 & 0.4000 & 0.3636 & 1 \\
$d_{2}$ & 2.8565 & 0.3952 & 0.3000 & 0.3571 & 2 \\
$d_{3}$ & 1.9192 & 0.2655 & 0.3000 & 0.2793 & 3 \\
\hline
\end{tabular}


Table 12. Scores of three candidates with three measures.

\begin{tabular}{ccccccc}
\hline Candidate & $\boldsymbol{s}_{\boldsymbol{P}}\left(\boldsymbol{\alpha}_{\boldsymbol{i}}\right)$ & Ranking & $\boldsymbol{s}_{\boldsymbol{E}}\left(\boldsymbol{\alpha}_{\boldsymbol{i}}\right)$ & Ranking & $\boldsymbol{s}_{\boldsymbol{H}}\left(\boldsymbol{\alpha}_{\boldsymbol{i}}\right)$ & Ranking \\
\hline$A_{1}$ & 0.3932 & 2 & 0.3781 & 2 & 0.3804 & 2 \\
$A_{2}$ & 0.4444 & 1 & 0.4339 & 1 & 0.4346 & 1 \\
$A_{3}$ & 0.3494 & 3 & 0.3601 & 3 & 0.3597 & 3 \\
\hline
\end{tabular}

Table 13. Resolutions of rankings based on three measures.

\begin{tabular}{ccccc}
\hline Measure & Large/medium & Medium/small & Overall resolution & Ranking \\
\hline Projection & 1.1302 & 1.1254 & 2.2556 & 1 \\
Euclid & 1.1476 & 1.0500 & 2.1976 & 3 \\
Hamming & 1.1425 & 1.0575 & 2.2000 & 2 \\
\hline
\end{tabular}

Table 13 shows that the projection is the best measure in the sense of overall resolution, followed by the Hamming distance and the Euclidean distance. That is to say, the projection-based method is a highresolution decision method.

\section{Conclusion and further research}

GDM is a very active research topic among all decision making methods. So far, as we know, the separation between decision objects has been measured by the distance measurement in most of the decision methods; the included angle between objects is often ignored. To overcome this drawback, this paper has introduced a projection measurement instead of the distance measurement to deal with the GDM problems. The main contribution of this paper is providing a novel GDM method, which can provide a ranking for a finite number of alternatives, which is high-resolution and robust.

The proposed method has some practical advantages. On the one hand, its idea is straightforward. The projection of an object on another one, in a sense, is an indication of its closeness to the other. Thus, it is easy to understand. On the other hand, its technology is comprehensive. The projection measurement considers not only the module size but also the included angle between two objects. Therefore, its considerations are more comprehensive. Moreover, its methodology is also comprehensive. The weight of DM is considered by its individual importance, uniform importance, and an optimistic coefficient; as a result, the proposed method is a comprehensive methodology. Finally, its procedure is simple. The projection-based GDM method is straightforward and can be implemented easily on a computer.

The proposed method has important theoretical and practical implications. On the one hand, the existing literature has paid very little attention to the development of projection measure, methodology, and technique. Thus, there is still room for improvement of
GDM. On the other hand, the proposed method not only can evaluate a real-life supplier evaluation and selection problem, but also may be used to evaluate the service quality and service performance, such as the performance of library service, the customer satisfaction in e-retailing, and the satisfaction and loyalty for smartphone users. Meanwhile, the proposed method is expected to be applicable to other areas such as risk investment and engineering management. Furthermore, since the proposed method is different from the existing methods, it may provide scholars with more methods for solving GDM problems. For example, the method proposed in this paper can be easily extended to solve the heterogeneous GDM problems.

In addition, we have only considered the IFN as a decision information in the decision process, which is a limitation. Other decision information, such as the ordinary fuzzy number and the interval-valued intuitionistic fuzzy number, may be left for our future study. In the future, we will continue working on the application of our work to other areas [65-68].

\section{Acknowledgments}

The authors would like to thank the editors and the anonymous reviewers for their insightful and constructive comments and suggestions that have led to this improved version of the paper.

\section{References}

1. Zavadskas, E., Antucheviciene, J., Turskis, Z. and Adeli, H. "Hybrid multiple-criteria decision-making methods: A review of applications in engineering", Scientia Iranica, 23(1), pp. 1-20 (2016).

2. Chen, T.-Y. "An interval type-2 fuzzy LINMAP method with approximate ideal solutions for multiple criteria decision analysis", Information Sciences, 297, pp. 50-79 (2015).

3. Chen, T.-Y. "An interval-valued intuitionistic fuzzy permutation method with likelihood-based preference 
functions and its application to multiple criteria decision analysis", Applied Soft Computing, 42, pp. 390409 (2016).

4. Liu, P. and Jin, F. "The trapezoid fuzzy linguistic bonferroni mean operators and their application to multiple attribute decision making", Scientia Iranica, 19(6), pp. 1947-1959 (2012).

5. Xu, J., Wan, S.-P. and Dong, J.-Y. "Aggregating decision information into Atanassov's intuitionistic fuzzy numbers for heterogeneous multi-attribute group decision making", Applied Soft Computing, 41, pp. 331-351 (2016).

6. Ureña, R., Chiclana, F., Morente-Molinera, J. and Herrera-Viedma, E. "Managing incomplete preference relations in decision making: A review and future trends", Information Sciences, 302, pp. 14-32 (2015).

7. Martı, L. and Herrera, F. "An overview on the 2-tuple linguistic model for computing with words in decision making: Extensions, applications and challenges", Information Sciences, 207, pp. 1-18 (2012).

8. Xu, Z. and Zhao, N. "Information fusion for intuitionistic fuzzy decision making: An overview", Information Fusion, 28, pp. 10-23 (2016).

9. Yue, Z. "TOPSIS-based group decision-making methodology in intuitionistic fuzzy setting", Information Sciences, 277, pp. 141-153 (2014).

10. Chen, X., Zhang, H. and Dong, Y. "The fusion process with heterogeneous preference structures in group decision making: A survey", Information Fusion, 24, pp. $72-83$ (2015).

11. Rodríguez, R., Martınez, L. and Herrera, F. "A group decision making model dealing with comparative linguistic expressions based on hesitant fuzzy linguistic term sets", Information Sciences, 241, pp. 28-42 (2013).

12. Chen, S., Cheng, S. and Chiou, C. "Fuzzy multiattribute group decision making based on intuitionistic fuzzy sets and evidential reasoning methodology", Information Fusion, 27, pp. 215-227 (2016).

13. Ebrahimnejad, S., Mousavi, S., TavakkoliMoghaddam, R., Hashemi, H. and Vahdani, B. "A novel two-phase group decision making approach for construction project selection in a fuzzy environment", Applied Mathematical Modelling, 36(9), pp. 4197-4217 (2012).

14. Vahdani, B., Mousavi, S.M., Hashemi, H., Mousakhani, M. and Tavakkoli-Moghaddam, R. "A new compromise solution method for fuzzy group decisionmaking problems with an application to the contractor selection", Engineering Applications of Artificial Intelligence, 26(2), pp. 779-788 (2013).

15. Mousavi, S.M., Jolai, F. and Tavakkoli-Moghaddam, R. "A fuzzy stochastic multi-attribute group decisionmaking approach for selection problems", Group Decision and Negotiation, 22(2), pp. 207-233 (2013).
16. Vahdani, B., Mousavi, S.M. and TavakkoliMoghaddam, R. "Group decision making based on novel fuzzy modified TOPSIS method", Applied Mathematical Modelling, 35(9), pp. 4257-4269 (2011).

17. Mousavi, S.M., Vahdani, B., Tavakkoli-Moghaddam, R. and Tajik, N. "Soft computing based on a fuzzy grey group compromise solution approach with an application to the selection problem of material handling equipment", International Journal of Computer Integrated Manufacturing, 27(6), pp. 547-569 (2014).

18. Mousavi, S.M., Torabi, S.A. and TavakkoliMoghaddam, R. "A hierarchical group decisionmaking approach for new product selection in a fuzzy environment", Arabian Journal for Science and Engineering, 38(11), pp. 3233-3248 (2013).

19. Sadr, S., Saroj, D., Kouchaki, S., Ilemobade, A. and Ouki, S. "A group decision-making tool for the application of membrane technologies in different water reuse scenarios", Journal of Environmental Management, 156, pp. 97-108 (2015).

20. Fasanghari, M., Amalnick, M., Anvari, R. and Razmi, J. "A novel credibility-based group decision making method for enterprise architecture scenario analysis using data envelopment analysis", Applied Soft Computing, 32, pp. 347-368 (2015).

21. Aloini, D., Dulmin, R. and Mininno, V. "A peer IFTOPSIS based decision support system for packaging machine selection", Expert Systems with Applications, 41(5), pp. 2157-2165 (2014).

22. Merigó, J., Casanovas, M. and Yang, J. "Group decision making with expertons and uncertain generalized probabilistic weighted aggregation operators", European Journal of Operational Research, 235(1), pp. 215-224 (2014).

23. Feng, B. and Lai, F. "Multi-attribute group decision making with aspirations: A case study", Omega, 44, pp. 136-147 (2014).

24. Srdjevic, B. and Srdjevic, Z. "Synthesis of individual best local priority vectors in AHP-group decision making", Applied Soft Computing, 13(4), pp. 20452056 (2013).

25. Tapia-Rosero, A., Bronselaer, A. and De Tré, G. "A method based on shape-similarity for detecting similar opinions in group decision-making", Information Sciences, 258, pp. 291-311 (2014).

26. Li, C., Zeng, S., Pan, T. and Zheng, L. "A method based on induced aggregation operators and distance measures to multiple attribute decision making under 2-tuple linguistic environment", Journal of Computer and System Sciences, 80(7), pp. 1339-1349 (2014).

27. Zhang, S. and Liu, S. "A GRA-based intuitionistic fuzzy multi-criteria group decision making method for personnel selection", Expert Systems with Applications, 38(9), pp. 11401-11405 (2011).

28. Atanassov, K. "Intuitionistic fuzzy sets", Fuzzy Sets and Systems, 20(1), pp. 87-96 (1986). 
29. Zadeh, L. "Fuzzy sets", Information and Control, 8(3), pp. 338-353 (1965).

30. Merigó, J., Gil-Lafuente, A. and Yager, R. "An overview of fuzzy research with bibliometric indicators", Applied Soft Computing, 27, pp. 420-433 (2015).

31. Xu, Z. and Cai, X. "Recent advances in intuitionistic fuzzy information aggregation", Fuzzy Optimization and Decision Making, 9(4), pp. 359-381 (2010).

32. Yu, D., Merigó, J. and Zhou, L. "Interval-valued multiplicative intuitionistic fuzzy preference relations", International Journal of Fuzzy Systems, 15(4), pp. 412-422 (2013).

33. Xu, Y., Wang, H. and Merigó, J. "Intuitionistic fuzzy Einstein Choquet integral operators for multiple attribute decision making", Technological and Economic Development of Economy, 20(2), pp. 227-253 (2014).

34. Xu, Z. and Liao, H. "A survey of approaches to decision making with intuitionistic fuzzy preference relations", Knowledge-Based Systems, 80, pp. 131-142 (2015).

35. Das, S. and Kar, S. "Group decision making in medical system: An intuitionistic fuzzy soft set approach", Applied Soft Computing, 24, pp. 196-211 (2014).

36. Hashemi, H., Bazargan, J. and Mousavi, S.M. "A compromise ratio method with an application to water resources management: An intuitionistic fuzzy set", Water Resources Management, 27(7), pp. 2029-2051, (2013).

37. Hashemi, H., Bazargan, J., Mousavi, S.M. and Vahdani, B. "An extended compromise ratio model with an application to reservoir flood control operation under an interval-valued intuitionistic fuzzy environment", Applied Mathematical Modelling, 38(14), pp. 34953511 (2014).

38. Mousavi, S.M., Vahdani, B. and Behzadi, S.S. "Designing a model of intuitionistic fuzzy VIKOR in multiattribute group decision-making problems", Iranian Journal of Fuzzy Systems, 13(1), pp. 45-65 (2016).

39. Mousavi, S.M., Mirdamadi, S., Siadat, A., Dantan, J. and Tavakkoli-Moghaddam, R. "An intuitionistic fuzzy grey model for selection problems with an application to the inspection planning in manufacturing firms", Engineering Applications of Artificial Intelligence, $\mathbf{3 9}$, pp. 157-167 (2015).

40. Liu, P. "Some generalized dependent aggregation operators with intuitionistic linguistic numbers and their application to group decision making", Journal of Computer and System Sciences, 79(1), pp. 131-143 (2013).

41. İntepe, G., Bozdag, E. and Koc, T. "The selection of technology forecasting method using a multi-criteria interval-valued intuitionistic fuzzy group decision making approach", Computers and Industrial Engineering, 65(2), pp. 277-285 (2013).

42. Yue, Z. and Jia, Y. "An application of soft computing technique in group decision making under intervalvalued intuitionistic fuzzy environment", Applied Soft Computing, 13(5), pp. 2490-2503 (2013).
43. Meng, F., Zhang, Q. and Cheng, H. "Approaches to multiple-criteria group decision making based on interval-valued intuitionistic fuzzy Choquet integral with respect to the generalized $\lambda$-shapley index", Knowledge-Based Systems, 37, pp. 237-249 (2013).

44. Wan, S. and Dong, J. "A possibility degree method for interval-valued intuitionistic fuzzy multi-attribute group decision making", Journal of Computer and System Sciences, 80(1), pp. 237-256 (2014).

45. Zhang, X. and Xu, Z. "Soft computing based on maximizing consensus and fuzzy TOPSIS approach to interval-valued intuitionistic fuzzy group decision making", Applied Soft Computing, 26, pp. 42-56 (2015).

46. Wan, S., Xu, G., Wang, F. and Dong, J. "A new method for Atanassov's interval-valued intuitionistic fuzzy MAGDM with incomplete attribute weight information", Information Sciences, 316, pp. 329-347 (2015).

47. Yue, Z. "An intuitionistic fuzzy projection-based approach for partner selection", Applied Mathematical Modelling, 37(23), pp. 9538-9551 (2013).

48. Yue, Z. and Jia, Y. "A group decision making model with hybrid intuitionistic fuzzy information", Computers \& Industrial Engineering, 87, pp. 202-212 (2015).

49. $\mathrm{Xu}, \mathrm{Z}$. and $\mathrm{Hu}, \mathrm{H}$. "Projection models for intuitionistic fuzzy multiple attribute decision making", International Journal of Information Technology \& Decision Making, 9(2), pp. 267-280 (2010).

50. Wei, G. "Decision-making based on projection for intuitionistic fuzzy multiple attributes", Chinese Journal of Management, 6(9), pp. 1154-1156 (2009).

51. Zheng, G., Jing, Y., Huang, H. and Gao, Y. "Application of improved grey relational projection method to evaluate sustainable building envelope performance", Applied Energy, 87(2), pp. 710-720 (2010).

52. Yue, Z. "Application of the projection method to determine weights of decision makers for group decision making", Scientia Iranica, 19(3), pp. 872-878 (2012).

53. Xu, G. and Liu, F. "An approach to group decision making based on interval multiplicative and fuzzy preference relations by using projection", Applied Mathematical Modelling, 37(6), pp. 3929-3943 (2013).

54. Xu, Z. and Da, Q. "Projection method for uncertain multi-attribute decision making with preference information on alternatives", International Journal of Information Technology \& Decision Making, 3(03), pp. 429-434 (2004).

55. Yue, Z. "Approach to group decision making based on determining the weights of experts by using projection method", Applied Mathematical Modelling, 36(7), pp. 2900-2910 (2012).

56. Yue, Z. and Jia, Y. "A direct projection-based group decision-making methodology with crisp values and interval data", Soft Computing, 21(9), pp. 2395-2405 (2017). 
57. Szmidt, E. and Kacprzyk, J. "Using intuitionistic fuzzy sets in group decision making", Control and Cybernetics, 31(4), pp. 1037-1054 (2002).

58. Shu, M., Cheng, C. and Chang, J. "Using intuitionistic fuzzy sets for fault-tree analysis on printed circuit board assembly", Microelectronics and Reliability, 46(12), pp. 2139-2148 (2006).

59. Boran, F., Kurt, M. and Akay, D. "A multi-criteria intuitionistic fuzzy group decision making for supplier selection with TOPSIS method", Expert Systems with Applications, 36(8), pp. 11363-11368 (2009).

60. Harsanyi, J. "Cardinal welfare, individualistic ethics, and interpersonal comparisons of utility", The Journal of Political Economy, 63(4), pp. 309-321 (1955).

61. Chen, S. and Tan, J. "Handling multicriteria fuzzy decision-making problems based on vague set theory", Fuzzy Sets and Systems, 67(2), pp. 163-172 (1994).

62. Hong, D. and Choi, C. "Multicriteria fuzzy decisionmaking problems based on vague set theory", Fuzzy Sets and Systems, 114(1), pp. 103-113 (2000).

63. Devi, K. "Extension of VIKOR method in intuitionistic fuzzy environment for robot selection", Expert Systems with Applications, 38(11), pp. 14163-14168 (2011).
64. Yue, Z. "An extended TOPSIS for determining weights of decision makers with interval numbers", KnowledgeBased Systems, 24(1), pp. 146-153 (2011).

65. Cebi, S. "Determining importance degrees of website design parameters based on interactions and types of websites", Decision Support Systems, 54(2), pp. 10301043 (2013).

\section{Biographies}

Zhongliang Yue was born in China in 1957. He is a Professor in the College of Science at Guangdong Ocean University. His current research interests include information fusion, group decision-making, fuzzy group decision-making, and their applications. He has contributed to over 70 journal articles.

Yuying Jia was born in China in 1959. She is an Associate Research Librarian in the Library at Guangdong Ocean University. Her current research interests focus on information and intelligence science, reader and user satisfaction, and professional collections. She has contributed to over 20 journal articles. 